$\underline{\text { Preprint typeset in JHEP style - PAPER VERSION }}$

March 2004

UB-ECM-PF-04/06

\title{
Matching Regge Theory to the OPE
}

\author{
S.S. Afonin*, A.A. Andrianov*, V.A. Andrianov* and D. Espriu $\diamond$ \\ *V.A. Fock Department of Theoretical Physics, St. Petersburg State University, \\ 1 ul.Ulianovskaya, 198504, Russia \\ $\diamond$ Departament d'Estructura i Constituents de la Matèria and CER for Astrophysics, \\ Particle Physics and Cosmology, Universitat de Barcelona, 647 Diagonal, 08028, Spain \\ E-mail: afonin24@mail.ru, andrianov@bo.infn.it, \\ Vladimir.Andrianov@pobox.spbu.ru, espriu@ecm.ub.es
}

\begin{abstract}
The spectra of masses and decay constants for non-strange meson resonances in the energy range $0-2.5 \mathrm{GeV}$ is analyzed. It is known from meson phenomenology that for given quantum numbers these spectra approximately follow linear trajectories with a universal slope. These facts can be understood in terms of an effective string description for QCD. For light meson states the trajectories deviate noticeably from the linear behavior. We investigate the possible corrections to the linear trajectories by matching two-point correlators of quark currents to the Operator Product Expansion (OPE). We find that the allowed modifications to the linear Regge behavior must decrease rapidly with the principal quantum number. After fitting the lightest states in each channel and certain low-energy constants the whole spectrum for meson masses and residues is obtained in a satisfactory agreement with phenomenology. We briefly speculate on possible implications for the QCD effective string.
\end{abstract}

KEYWORDS: qcd,nex,sru,pmo. 


\section{Contents}

1. Introduction 1

2. Current-current correlators and scheme of sum rules 3

3. Linear trajectories for vector and axial-vector resonances 4

4. Sum rules and deviation from the LTA in the $V, A$ channels $\quad 7$

5. Sum rules for scalar and pseudoscalar resonances 10

6. Details of fits and results 11

$\begin{array}{ll}\text { 7. Summary } & 14\end{array}$

A. Note on the role of dimension-two condensate 15

$\begin{array}{lr}\text { B. Sum rules } & 16\end{array}$

$\begin{array}{ll}\text { C. Reference formulae } & 18\end{array}$

D. D-wave vector mesons $\quad 19$

E. Electromagnetic pion mass difference $\Delta m_{\pi}$ and chiral constants $L_{8}, L_{10} \cdot 19$

$\begin{array}{ll}\text { F. Numerical analysis } & 21\end{array}$

\section{Introduction}

Hadron phenomenology tells us that the masses squared of mesons with given quantum numbers lie approximately on equally-spaced linear trajectories ${ }^{1}$ (see, for example, the modern reviews $[1,2])$. This is a strong indication that QCD admits an effective string description, as this type of spectrum is characteristic e.g. of the bosonic ${ }^{2}$ string [3]. Long ago it was noticed [5] that if one accepts this behavior, by including an infinite number of resonances it is possible to reproduce the parton-model logarithm present in two-point correlators.

\footnotetext{
${ }^{1}$ From now on, we shall term this behavior "linear trajectory ansatz" (LTA).

${ }^{2}$ This string model is of course not consistent, a more realistic amplitude is the Lovelace-Shapiro amplitude but unfortunately it cannot be derived from any known string and as we shall see is also incompatible with QCD anyway. See e.g. [4] for a discussion on this point.
} 
This observation means that the exchange of an infinite number of vector mesons may be dual to the perturbative QCD continuum made of quarks and gluons. This quarkhadron duality was explicitly verified in QCD in $1+1$ dimensions in the large- $N_{c}$ limit $[6,7]$. At present, the QCD community understand by quark-hadron duality several, somewhat different, things. The most extended assertion is that perturbation theory can be used to calculate a certain smeared hadronic cross section (so-called global duality) [8]. We use the term in a rather lax sense and understand by quark-hadron duality any matching of hadronic and partonic degrees of freedom.

In the context of large- $N_{c} \mathrm{QCD}$, quark-hadron duality for mesons has received a lot of attention [9]- [23]. In [9] the parameters of linear vector mass spectrum were derived within the framework of finite-energy sum rules. The axial-vector channel was also considered there. Later, this approach was extended to the pseudoscalar [10] and scalar [11] cases. In [13] the problem of matching this infinite set of meson states to the Operator Product Expansion (OPE) [14] was considered, with $m_{V}^{2}(n)$ being a general function. In the review [15] possible deviations from quark-hadron duality were investigated (including meson resonance widths, i.e. away from the large- $N_{c}$ limit.) In [16] the linear trajectory ansatz in the vector and axial channels were used to calculate certain physical observables. Subsequently, in [17]- [23] various aspects of quark-hadron duality, chiral symmetry restoration (CSR), and matching to the OPE have been discussed.

Since, after all, it is well known that the linear trajectory ansatz fails to describe the lightest states in each channel (particularly so in the pseudoscalar channel), some attempts to go beyond the linear trajectory ansatz were studied in $[18,19]$, in the latter paper using a semi-classical string analysis. The ansätze proposed there, however, seem to lead to a discrepancy with the OPE that will be explained later.

In the present work we want to re-take these issues. Our interest has, to some extent, been triggered by the recent controversy among the authors of references [17] and [21], but we shall also report here on some older results of us [22]. We shall consider radial Regge trajectories for meson resonances with the same quantum numbers (so-called "radial excitations") in the vector $(V)$, axial-vector $(A)$, scalar $(S)$, and pseudoscalar $(P)$ correlation functions. If one believes in the existence of a QCD effective string - and we do - the slope of trajectories must be the same in all channels because this slope is proportional to the string tension that depends on gluodynamics only (string universality). In this respect we tend to agree with the approach of [17], but we find other aspects of this work to be more questionable.

We propose a systematic method to take into account possible corrections to the linear trajectories in the $V, A, S, P$ channels. Our ansatz for the meson mass spectra, once substituted into two-point correlators, is matched to the OPE, which gives a set of constraints on meson mass parameters. As we shall see, the OPE dictates a very particular class of corrections to the linear trajectory ansatz. These corrections are not anecdotic: they turn out to be essential to get good phenomenology and by taking them into account one does not need to introduce an artificial separation between low-lying and excited radial excitations in the different channels like in $[16,17,20,21]$. Finally, the results are fitted to the experimental data $[1,2,24]$. 
The paper is organized as follows. In section 2 we remind the general idea of how to construct the asymptotic sum rules for the case of infinite number of resonances. In particular, the status and the necessity of non-linear corrections to the linear trajectory ansatz is thoroughly examined in sections 3 and 4 for the case of $V, A$ mesons. In section 5 this analysis is extended to the $S, P$ channels. Section 6 contains the details of our fits and discussion of the obtained results. In section 7 we outline our conclusions.

\section{Current-current correlators and scheme of sum rules}

Let us consider the two-point correlators of $V, A, S, P$ quark currents in the large- $N_{c}$ limit of QCD. On the one hand, by virtue of confinement they are saturated by an infinite set of narrow meson resonances, that is, they can be represented by the sum of narrow meson resonances with given quantum numbers $J$ and masses $m_{J}(n), n=0,1, \ldots$,

$$
\begin{gathered}
\Pi^{J}\left(Q^{2}\right)=\int d^{4} x \exp (i Q x)\langle\bar{q} \Gamma q(x) \bar{q} \Gamma q(0)\rangle_{\text {planar }}=\sum_{n} \frac{Z_{J}(n)}{Q^{2}+m_{J}^{2}(n)}+D_{0}^{J}+D_{1}^{J} Q^{2}, \\
J \equiv S, P, V, A ; \quad \Gamma=i, \gamma_{5}, \gamma_{\mu}, \gamma_{\mu} \gamma_{5} ; \quad D_{0}, D_{1}=\text { const. }
\end{gathered}
$$

The last two terms both in the $S, P$ and in the $V, A$ channels represent a perturbative contribution, with $D_{0}$ and $D_{1}$ being contact terms required to eliminate divergences in the ultraviolet. On the other hand, their high-energy asymptotics is provided [14,25] by perturbation theory and the OPE. In the chiral limit ${ }^{3}$

$$
\begin{gathered}
\Pi^{V}\left(Q^{2}\right)=\frac{1}{4 \pi^{2}}\left(1+\frac{\alpha_{s}}{\pi}\right) \ln \frac{\mu^{2}}{Q^{2}}+\frac{\alpha_{s}}{12 \pi} \cdot \frac{\left\langle\left(G_{\mu \nu}^{a}\right)^{2}\right\rangle}{Q^{4}}-\frac{28}{9} \pi \alpha_{s} \frac{\langle\bar{q} q\rangle^{2}}{Q^{6}}, \\
\Pi^{A}\left(Q^{2}\right)=\frac{1}{4 \pi^{2}}\left(1+\frac{\alpha_{s}}{\pi}\right) \ln \frac{\mu^{2}}{Q^{2}}+\frac{\alpha_{s}}{12 \pi} \cdot \frac{\left\langle\left(G_{\mu \nu}^{a}\right)^{2}\right\rangle}{Q^{4}}+\frac{44}{9} \pi \alpha_{s} \frac{\langle\bar{q} q\rangle^{2}}{Q^{6}} \\
\Pi^{S}\left(Q^{2}\right)=-\frac{3}{8 \pi^{2}}\left(1+\frac{11 \alpha_{s}}{3 \pi}\right) Q^{2} \ln \frac{\mu^{2}}{Q^{2}}+\frac{\alpha_{s}}{8 \pi} \cdot \frac{\left\langle\left(G_{\mu \nu}^{a}\right)^{2}\right\rangle}{Q^{2}}-\frac{22}{3} \pi \alpha_{s} \frac{\langle\bar{q} q\rangle^{2}}{Q^{4}} \\
\Pi^{P}\left(Q^{2}\right)=-\frac{3}{8 \pi^{2}}\left(1+\frac{11 \alpha_{s}}{3 \pi}\right) Q^{2} \ln \frac{\mu^{2}}{Q^{2}}+\frac{\alpha_{s}}{8 \pi} \cdot \frac{\left\langle\left(G_{\mu \nu}^{a}\right)^{2}\right\rangle}{Q^{2}}+\frac{14}{3} \pi \alpha_{s} \frac{\langle\bar{q} q\rangle^{2}}{Q^{4}}
\end{gathered}
$$

where we have defined

$$
\Pi_{\mu \nu}^{V, A}\left(Q^{2}\right) \equiv\left(-\delta_{\mu \nu} Q^{2}+Q_{\mu} Q_{\nu}\right) \Pi^{V, A}\left(Q^{2}\right)
$$

The vacuum saturation for the 4 -fermion condensate [14] in the large- $N_{c}$ limit has been used. In Eqs. (2.3)-(2.6) $\left\langle\left(G_{\mu \nu}^{a}\right)^{2}\right\rangle$ and the $\langle\bar{q} q\rangle$ represent the gluon and the quark condensate, respectively. The normalization scale $\mu$ arises after additive renormalization of the

\footnotetext{
${ }^{3}$ In [26] asymptotic terms governed by the dimension-two gluon condensate $\lambda^{2}$ ("gluon mass") were introduced. This dimension-two condensate cannot be produced by a local gauge-invariant operator [27,28]. On the other hand, this condensate brings little influence on the fits of meson parameters (see the discussion in Appendix A). We put here this condensate to zero.
} 
infinite sums in Eq. (2.1). $\alpha_{s}$ is the QCD coupling constant taken at the Chiral Symmetry Breaking $(\mathrm{CSB})$ scale $\sim 1 \mathrm{GeV}$.

As it can be seen from Eqs. (2.3)-(2.6) differences of correlators of opposite parity decrease very rapidly at large momenta

$$
\begin{aligned}
& \Pi^{V}\left(Q^{2}\right)-\Pi^{A}\left(Q^{2}\right)=-8 \pi \alpha_{s} \frac{\langle\bar{q} q\rangle^{2}}{Q^{6}}+\mathcal{O}\left(\frac{1}{Q^{8}}\right), \\
& \Pi^{S}\left(Q^{2}\right)-\Pi^{P}\left(Q^{2}\right)=-12 \pi \alpha_{s} \frac{\langle\bar{q} q\rangle^{2}}{Q^{4}}+\mathcal{O}\left(\frac{1}{Q^{6}}\right) .
\end{aligned}
$$

This signifies the chiral symmetry restoration (CSR) at high energies, with the quark condensate $\langle\bar{q} q\rangle$ being an order parameter of the chiral symmetry breaking in QCD.

Expanding the right-hand side of Eq. (2.1) in inverse powers of $Q^{2}$ and comparing with Eqs. (2.3)-(2.6) one obtains several asymptotic sum rules for each channel (we do not account for condensates of dimension eight and higher). If one deals with a finite number of resonances the procedure is straightforward (for the CSR sum rules [29,30]) . In the case of infinite number of resonances and given an ansatz $m^{2}(n)$ one uses the Euler-Maclaurin summation formula or, alternatively, the $\psi$-function (both methods are equivalent). It should be noticed here that, generally speaking, applying a summation procedure for a divergent sum and expanding in $1 / Q^{2}$ are not commutative operations. A regulator is in general called for.

The sum rules, obtained in this way, deliver certain constraints on the parameters of meson mass spectra. This information can be extracted and compared with phenomenology, which is the subject of the next sections.

In this work we shall ignore the anomalous dimensions of the operators and the running of the coupling constant, and therefore we do not take into account the logarithmic corrections that they may introduce in the sum rules. We shall come back to this issue in a separate paper.

\section{Linear trajectories for vector and axial-vector resonances}

In the $V, A$ case the residues $Z_{V, A}(n)$ in Eq. (2.1) have the structure

$$
Z_{V, A}(n)=2 F_{V, A}^{2}(n)
$$

where the quantities $F_{V, A}(n)$ represent the decay constants parameterizing the matrix elements of the corresponding currents

$$
\begin{aligned}
\left\langle 0\left|j_{\mathrm{em}}^{\mu}(0)\right| V(\epsilon, k, n)\right\rangle & =\frac{1}{(2 \pi)^{\frac{3}{2}}} e F_{V}(n) m_{V}(n) \epsilon^{\mu}, \\
\left\langle 0\left|A^{\mu}(0)\right| A(\epsilon, k, n)\right\rangle & =\frac{1}{(2 \pi)^{\frac{3}{2}}} e F_{A}(n) m_{A}(n) \epsilon^{\mu} .
\end{aligned}
$$

where $k$ is the meson momentum, $\epsilon^{\mu}$ is a polarization vector, and $e$ is electron charge. The quantity $F_{V}(n)$ can be obtained from the decay of vector mesons

$$
\Gamma_{V \rightarrow e^{+} e^{-}}(n)=\frac{4 \pi \alpha^{2} F_{V}^{2}(n)}{3 m_{V}(n)},
$$


where $\alpha$ is the fine structure constant. The constants $F_{A}(n)$ are related to the widths of some radiative decays. Unfortunately, this sector is poorly known, except for the reactions including the ground axial-vector state $\left(a_{1}\right.$-meson). We have found two examples $[31,32]$

$$
\Gamma_{\tau \rightarrow a_{1} \nu_{\tau}}=\frac{G_{F}^{2} m_{\tau}^{3} F_{a_{1}}^{2}}{16 \pi}\left(1-\frac{m_{a_{1}}^{2}}{m_{\tau}^{2}}\right)\left(1+\frac{2 m_{a_{1}}^{2}}{m_{\tau}^{2}}\right)
$$

and

$$
\Gamma_{a_{1} \rightarrow \pi \gamma}=\frac{\alpha F_{a_{1}}^{2} m_{a_{1}}}{24 f_{\pi}^{2}}\left(1-\frac{m_{\pi}^{2}}{m_{a_{1}}^{2}}\right)^{3} .
$$

where $G_{F}$ is the Fermi constant, $m_{\tau}$ is the mass of the $\tau$-lepton, and $f_{\pi}$ is the pion decay constant.

Let us now introduce the linear trajectory ansatz for the meson mass spectrum

$$
m_{V, A}^{2}(n)=M_{V, A}^{2}+a_{V, A} n ; \quad F_{V, A}^{2}(n)=\text { const } \equiv F_{V, A}^{2} ; \quad n=0,1,2, \ldots,
$$

with constant intercepts $M_{V, A}^{2}$ and slopes $a_{V, A}$. In order to do the sums over all resonances we shall use the Euler-Maclaurin summation formula in Eq. (2.1)

$$
\begin{gathered}
\sum_{n=0}^{N} f(n)=\int_{0}^{N} f(x) d x+\frac{1}{2}[f(0)+f(N)]+\frac{B_{1}}{2 !}\left[f^{\prime}(N)-f^{\prime}(0)\right]- \\
\frac{B_{2}}{4 !}\left[f^{\prime \prime \prime}(N)-f^{\prime \prime \prime}(0)\right]+\ldots+(-1)^{k} \frac{B_{k+1}}{(2 k+2) !}\left[f^{(2 k+1)}(N)-f^{(2 k+1)}(0)\right]+\ldots,
\end{gathered}
$$

where $B_{1}=1 / 6, B_{2}=1 / 30, \ldots$ are Bernoulli numbers. We shall expand the final expression in powers of $Q^{-2}$ and after comparing with the OPE's (2.3) and (2.4) one gets the set of asymptotic sum rules.

At this point we have to introduce a regulator ${ }^{4}$ since the series is manifestly not absolutely convergent (the general term behaves like $\sim 1 / n$ ). We shall cut-off the infinite sum by including only the $N$ first terms in the sum. This naturally cuts off the restricted value of momenta to $\Lambda_{\mathrm{cut} ; \mathrm{V}, \mathrm{A}}^{2} \equiv M_{V, A}^{2}+a_{V, A} N_{V, A} \simeq a_{V, A} N_{V, A}$. As we identify paritydoublers between the $V$ - and $A$-channels and do not intent the cutoff to break chiral symmetry, the cutoff numbers $N_{V}$ and $N_{A}$ must be taken equal, $N_{V}=N_{A}$. On the other hand, the appropriate cutoffs in the OPE also coincide in the $V, A$-channels in a chirally symmetric regularization ${ }^{5}$, so $\Lambda_{\text {cut; } \mathrm{V}}=\Lambda_{\text {cut;A }}$ (see also similar arguments in [18]). Therefore for a natural implementation of chiral symmetry the slopes of radial Regge trajectories must be the same, $a_{V}=a_{A}$. To compare with OPE (2.3) and (2.4) one has to make an additive renormalization by means of subtraction of infinite constants $D_{0}^{V, A}$. In order not to break

\footnotetext{
${ }^{4}$ Another way to improve the convergence is to differentiate with respect to $Q^{2}$. This recipe will be used below for the matching to QCD.

${ }^{5}$ Note that a momentum cut-off is questionable in the OPE expansions on account of gauge invariance , but perfectly gauge invariant in the sum over resonances (2.1). So one is actually forced to use different regulators on both sides, but after the renormalization with the help of constants $D_{0}^{V, A}$ the leading logarithmic term in $\mu^{2}$ is in any case unambiguous.
} 
the chiral symmetry the subtraction must be equal for parity doublers, $D_{0}^{V}=D_{0}^{A}$. The conclusion is then

$$
\frac{4}{3} \cdot \frac{N_{c}}{16 \pi^{2}}=\frac{2 F_{V}^{2}}{a_{V}}=\frac{2 F_{A}^{2}}{a_{A}}
$$

We remark that the QCD string model implies $a_{V}=a_{A} \equiv a$ as well. (This relation $a_{V}=a_{A}$ is not fulfilled in $[16,20]$ where such analysis for the LTA was performed.) Then one concludes that $F_{V}=F_{A}$. Note that the limit $N \rightarrow \infty$, or equivalently, $\Lambda_{\text {cut }} \rightarrow \infty$ is assumed. This allows us to neglect terms of the form $Q^{2} / \Lambda_{\text {cut }}^{2}$ or $m^{2} / \Lambda_{\text {cut }}^{2}$.

However, as we have already mentioned, it is not possible to extract further consequences from the individual $V$ and $A$ asymptotic sum rules. Sub-leading terms are somewhat ambiguous. Fortunately, the annoying logarithm is absent in differences of correlation functions. Namely

$$
\Pi^{V}\left(Q^{2}\right)-\Pi^{A}\left(Q^{2}\right)=2 \sum_{n=0}^{\infty}\left(\frac{F_{V}^{2}(n)}{Q^{2}+m_{V}^{2}(n)}-\frac{F_{A}^{2}(n)}{Q^{2}+m_{A}^{2}(n)}\right)
$$

This difference (and the equivalent for $S, P$ ) show a very fast CSR. The leading contribution is of $\mathcal{O}\left(1 / Q^{6}\right)$ : all perturbative and purely gluonic contributions cancel in the difference. For individual sum rules we shall perform the appropriate derivatives in $Q^{2}$ before matching OPE and radial Regge trajectories.

The above fast convergence in correlator differences was used among others by [17], where the following generalization of the Weinberg sum rules [33] was proposed

$$
\begin{aligned}
\sum_{n=0}^{\infty}\left(F_{V}^{2}(n) m_{V}^{2 i}(n)-F_{A}^{2}(n) m_{A}^{2 i}(n)\right) & =C^{(i)}, \quad i=0,1 \\
C^{(0)}=f_{\pi}^{2}, \quad C^{(1)} & =0 .
\end{aligned}
$$

We emphasize that one sums over chiral pairs in the difference (3.11). This becomes very important at the moment that one needs to cut-off the sums with a finite value of $N$. If the cut-off is placed in such a way that the "chiral partner" of a given resonance, that is included, is left out, chiral symmetry will be explicitly broken by the regulator. It is thus vital to properly identify "chiral partners". This is not an issue in the $V, A$ cases, but it will be so as we shall see in the $S, P$ channels.

From the second sum rule $(i=1)$ it immediately follows, given that $F_{A}=F_{V}$ and $a_{A}=a_{V}$, that $M_{A}=M_{V}$. This automatically implies if the believe in the LTA that the spectrum of QCD is degenerate in the vector and axial-vector channels, something that it is obviously not true (even after correcting for the quark mass differences, not included in the present analysis). Furthermore, the first sum rule $(i=0)$, implies $f_{\pi}=0$, something again manifestly incompatible with experimental evidence. Since $f_{\pi}$ can be interpreted as the order parameter of chiral symmetry, what we are seeing here is that the LTA corresponds to a string model that has many common features with QCD, but an essential one is missing, 
namely it would correspond to a string model without chiral symmetry breaking ${ }^{6}$. Note that convergence of the infinite sums in Eq. (3.11) also requires to impose the equality of slopes $a_{V}=a_{A}$, intercepts $M_{V}=M_{A}$, and decay constants $F_{V}=F_{A}$.

Of course one may escape the contradiction between the fact that the LTA leads to $f_{\pi}=0$ and the real world by declaring that the ground states (perhaps even the first few resonances) in each channel, e.g. the $\rho$ and $a_{1}$ mesons, are isolated resonances. This pattern roughly meets the existing spectroscopy of $V, A$ mesons, but it does not reproduce the physical values of condensates [16]. However, such an ansatz satisfies the CSR (3.11) at high energies. Obviously this way out is quite ad-hoc and cannot be justified on any QCD-inspired string model.

In the present paper we want to argue that the ansätze proposed in $[16,17]$ can be improved by introducing certain systematic corrections to the linear trajectories (3.7). The problem here is that arbitrary ansätze for $m^{2}(n)$ and $F^{2}(n)$ result in appearance of terms which are absent in the standard OPE, namely, terms with a fractional power of $Q^{2}$ and terms of the kind $Q^{-2 k} \ln \frac{\Lambda_{\text {cut }}^{2}}{Q^{2}}$. (Recall that we do not consider anomalous dimensions.)

\section{Sum rules and deviation from the LTA in the $V, A$ channels}

Our goal is to construct a class of radial Regge trajectories that does not lead to the unwanted terms. Namely, our ansatz must reproduce the parton-model logarithm and contain only inverse powers of large momentum squared $Q^{2}$ besides the logarithm. Recall that, using (3.8),

$$
\sum_{n=0}^{N} \frac{F^{2}(n)}{Q^{2}+m^{2}(n)}=\int_{0}^{N} \frac{F^{2}(x) d x}{Q^{2}+m^{2}(x)}+\mathcal{O}\left(\frac{1}{Q^{2}}\right) .
$$

Clearly the logarithm can be only produced by the integral. In order to generate the logarithm we require that

$$
\int \frac{F^{2}(x) d x}{Q^{2}+m^{2}(x)}+D_{0}=C \ln \left(\frac{Q^{2}+m^{2}(x)}{\mu^{2}}\right)+\mathcal{O}\left(\frac{1}{Q^{2}}\right),
$$

here $C$ is a constant from $\mathrm{OPE}(3)$ and (4), $D_{0}$ is a subtraction constant, and $\mu$ is a normalization scale.

Differentiating Eq. (4.2) with respect to $x$, one can see that the two requirements are satisfied only if

$$
F^{2}(x)=t(x) \frac{d m^{2}(x)}{d x}, \quad t(x)=C+\Delta t(x)
$$

\footnotetext{
${ }^{6}$ The Lovelace-Shapiro amplitude, after correcting the intercept has the proper Adler zero, required by chiral symmetry. However, it still has regularly spaced levels, something that as we see is manifestly incompatible with QCD asymptotics. It cannot be the correct QCD string. The interested reader may want to see [4] for an attempt to formulate consistent string propagation in a background where chiral symmetry has been broken.
} 
where $\Delta t(x)$ is a decreasing function to be defined. If we do not consider anomalous dimensions the function $\Delta t(x)$ does not have to induce any logarithms and other nonpolynomial in $Q^{-2}$ terms in the integral (4.2). Thus, the direct expansion of the integral

$$
\int_{0}^{m^{2}(N)} \frac{\Delta t(x) d\left(m^{2}(x)\right)}{Q^{2}+m^{2}(x)}=\sum_{k>0} t_{k}\left(\frac{1}{Q^{2}}\right)^{k},
$$

where

$$
t_{k}=\int_{0}^{m^{2}(N)} \Delta t(x)\left(-m^{2}(x)\right)^{k-1} d\left(m^{2}(x)\right),
$$

must exist at any order.

Convergence of the integrals in (4.4) for any $k$ is possible if the function $\Delta t(x)$ falls off as an exponential, or faster. Therefore, since one does not have yet any dynamical arguments let us take the simplest possibility, namely

$$
\Delta t(x)=A_{F} e^{-B_{F} x}, \quad B_{F}>0,
$$

with constant $A_{F}$ and $B_{F}$. Given an ansatz for $m^{2}(n)$, Eq. (4.3) together with (4.5) provides the condition of consistency with the OPE. It is clear that the LTA ansatz (3.7) is a simple particular case of Eq. (4.3). Any improvement of the ansatz (3.7) has to meet Eq. (4.3) as well. We remark that the corrections to linear trajectories, proposed in $[18,19]$, do not satisfy this requirement. Thus, these ansätze cannot be matched to the OPE.

The sum rules (3.11) can actually be generalized to values of $i$ greater than 1

$$
\sum_{n=0}^{\infty}\left(F_{V}^{2}(n) m_{V}^{2 i}(n)-F_{A}^{2}(n) m_{A}^{2 i}(n)\right)=C^{(i)}, \quad i=0,1, \ldots
$$

Now the $C^{(i)}$ contain a contribution from higher dimensional condensates. For the absolute convergence of Eq. (4.6) at a given $i$ one has to have

$$
\begin{array}{ll}
F_{V}^{2}(n)-F_{A}^{2}(n) \sim \frac{1}{n^{1+\alpha}}, & \alpha>i, \\
m_{V}^{2}(n)-m_{A}^{2}(n) \sim \frac{1}{n^{\beta}}, & \beta>i .
\end{array}
$$

Let us now discuss corrections to Eq. (3.7)

$$
m_{V, A}^{2}(n)=M_{V, A}^{2}+a n+\delta_{V, A}(n) .
$$

Note that the convergence for any $i$ requires $M_{V}=M_{A} \equiv M$ and that the contribution from the function $\delta_{V, A}(n)$ to the difference (4.8) must decrease at least exponentially.

Collecting our observations, we propose the simplest ansatz satisfying all previous requirements, keeping of course in mind that this is just one of the possibilities

$$
\begin{aligned}
m_{V, A}^{2}(n) & =M^{2}+a n+A_{m}^{V, A} e^{-B_{m} n}, \\
F_{V, A}^{2}(n) & =a\left(C+A_{F}^{V, A} e^{-B_{F} n}\right), \\
C & =\frac{1}{8 \pi^{2}}\left(1+\frac{\alpha_{s}}{\pi}\right), \quad B_{m}>0, \quad B_{F}>0,
\end{aligned}
$$


with certain constants $A_{m, F}^{V, A}, B_{m, F}$ to be fitted. We do not know the underlying dynamics responsible for the appearance of those exponential corrections. But it seems for us reasonable to suppose that, for masses, this dynamics is governed mostly by gluons and thereby does not depend on flavor. Thus, we keep the exponent $B_{m}$ the same for all channels. For the same reason we regard $B_{F}$ as independent of parity. We note also that in Eq. (4.3) it is enough to retain only the linear in $n$ part of $m^{2}(n)$.

In what follows we adopt a perturbative approach and retain in the sum rules only terms linear in the exponentially small corrections. Products of exponentials exceed the precision of our simple (one-exponential) parameterization: these products are regarded as of the order of next-to-next-to-leading corrections to Eqs. (4.10) and (4.11). One should keep in mind the precision of our approach and thus, when comparing the OPE and the sums of resonances, we only match the perturbative logarithms and the first non-perturbative contribution $\mathcal{O}\left(1 / Q^{4}\right)$ in the $V, A$ channels separately, and the leading (non-perturbative) correction $\mathcal{O}\left(1 / Q^{6}\right)$ in the difference $\Pi^{V}\left(Q^{2}\right)-\Pi^{A}\left(Q^{2}\right)$ which represents a true order parameter of the chiral symmetry breaking in the chiral limit.

In order to avoid of irrelevant infinite constant let us consider the first derivative of $V, A$-correlators. Introducing the notation for the linear part,

$$
\bar{m}^{2}(n) \equiv M^{2}+a n,
$$

and making use of (3.1) and (4.10)-(4.12) we have (the indices $V, A$, are dropped for brevity)

$$
\frac{d \Pi\left(Q^{2}\right)}{d Q^{2}}=-2 \sum_{n=0}^{\infty} \frac{a\left(C+A_{F} e^{-B_{F} n}\right)}{\left(Q^{2}+\bar{m}^{2}(n)+A_{m} e^{-B_{m} n}\right)^{2}} .
$$

For the ground states the exponential corrections are not small (in general, about 50\%). We will not apply our perturbative approach for these mesons. Separating out the ground states and retaining in the remainder only the parts linear in exponential corrections, one has for (4.14)

$$
\begin{aligned}
-\frac{1}{2} \frac{d \Pi\left(Q^{2}\right)}{d Q^{2}} & \simeq \frac{a\left(C+A_{F}\right)}{\left(Q^{2}+M^{2}+A_{m}\right)^{2}} \\
& \quad+\sum_{n=1}^{\infty}\left\{\frac{a C}{\left(Q^{2}+\bar{m}^{2}(n)\right)^{2}}+\frac{a A_{F} e^{-B_{F} n}}{\left(Q^{2}+\bar{m}^{2}(n)\right)^{2}}-\frac{2 a C A_{m} e^{-B_{m} n}}{\left(Q^{2}+\bar{m}^{2}(n)\right)^{3}}\right\}
\end{aligned}
$$

In the r.h.s. of Eq. (4.15) one has three sums. The first one represents the first derivative of $\psi$-function, which has the standard asymptotic expansion at large $Q^{2}$. In the second and third sums one may permute the summation over $n$ and expansion in $Q^{-2}$ due to absolute convergence in any order of this expansion. The final expressions for the sum rules are presented in Appendices B and C. (see Eqs. (B.3)-(B.7)).

The solution of these equations will be considered after discussion of the scalar sector. In addition, the so-called D-wave vector mesons should be taken into account, see Appendix D.

As a further evidence for the need of nonlinear corrections let us consider the chiral symmetry restoration limit $\langle\bar{q} q\rangle \rightarrow 0$ and $f_{\pi} \rightarrow 0$. As follows from Eqs. (B.3),(B.4), in 
the case of LTA the corresponding solution $M^{2}=\frac{1}{2} a$ gives a negative value for the gluon condensate in Eqs. (B.5), (B.6). This implies instability of vacuum energy [34] and, hence, the appearance of tachyons. In that sense the LTA is inconsistent with rather general principles, even in the chiral symmetry restoration limit. A consistent ansatz can be only non-linear.

\section{Sum rules for scalar and pseudoscalar resonances}

The scalar case differs from the vector one and the derivation presented in the previous section needs to be revisited. Due to the transversality (2.7), the $V, A$ correlators required only one subtraction in Eq. (2.1). The $S, P$ correlators require two subtractions. Accepting the definition ${ }^{7}$

$$
Z_{S, P}(n) \equiv 2 G_{S, P}^{2}(n) m_{S, P}^{2}(n),
$$

the analog of (4.2) in the $S, P$ case takes the form

$$
\int \frac{G^{2}(x) m^{2}(x) d x}{Q^{2}+m^{2}(x)}+D_{0}+D_{1} Q^{2}=-\bar{C} Q^{2} \ln \left(\frac{Q^{2}+m^{2}(x)}{\mu^{2}}\right)+\ldots,
$$

where $\bar{C}>0$, and $D_{0}, D_{1}$ are subtraction constants. Let us make the following rearrangement. The sum over resonances can be rewritten as

$$
\begin{aligned}
\Pi\left(Q^{2}\right)=2 \sum_{n} \frac{G^{2}(n) m^{2}(n)}{Q^{2}+m^{2}(n)} & +D_{0}+D_{1} Q^{2} \\
& =\left[\sum_{n} 2 G^{2}(n)+D_{0}\right]-Q^{2}\left[\sum_{n} \frac{2 G^{2}(n)}{Q^{2}+m^{2}(n)}-D_{1}\right] .
\end{aligned}
$$

The first sum in the right-hand side of (5.3) represents an infinite constant and it must be renormalized in a chirally symmetric way

$$
\sum_{n} 2 G^{2}(n)+D_{0}=\tilde{D}_{0}
$$

where $\tilde{D}_{0}$ is different for the $S$ and $P$ channels. Then we have

$$
\int \frac{Q^{2} G^{2}(x) d x}{Q^{2}+m^{2}(x)}-\frac{1}{2} D_{1} Q^{2}=\bar{C} Q^{2} \ln \left(\frac{Q^{2}+m^{2}(x)}{\mu^{2}}\right)+\ldots
$$

Repeating the discussion in the previous section, which has led to Eq. (4.3), one obtains in the scalar case

$$
G_{S, P}^{2}(n)=a\left(\bar{C}+A_{G}^{S, P} e^{-B_{G} n}\right), \quad B_{G}>0,
$$

\footnotetext{
${ }^{7}$ This formula does not work for the $\pi$-meson if we include the pion in the radial Regge trajectory. Thus for the corresponding residue we just accept its value in the current algebra: $Z_{\pi}=2 \frac{\langle\bar{q} q\rangle}{f_{\pi}^{2}}$. As we anyway separate out the lowest state, this does not affect the subsequent analysis. Alternatively one could extend Eq. (5.1) with the help of a constant shift for all resonances in $Z_{S, P}(n)$ by a quantity $Z_{\pi}$. However this modification induces an unacceptably large value of dimension-two condensate in the OPE (see discussion in Appendix A). In accordance to known theoretical and phenomenological estimations $[26,27,36]$ we put this condensate to zero.
} 
where

$$
\bar{C}=\frac{3}{16 \pi^{2}}\left(1+\frac{11 \alpha_{s}}{3 \pi}\right) .
$$

Following the same arguments concerning the parameter $B_{m}$, as in the previous section, we propose the following parameterization of $S, P$ masses

$$
m_{S, P}^{2}(n)=\bar{M}^{2}+a n+A_{m}^{S, P} e^{-B_{m} n} .
$$

Now let us derive the asymptotic sum rules. In order to avoid irrelevant infinite terms we consider the second derivative of $S, P$-correlators. Making use of (4.13), (5.1), (5.6), and (5.8) we can write

$$
\frac{d^{2} \Pi\left(Q^{2}\right)}{\left(d Q^{2}\right)^{2}}=4 \sum_{n=0}^{\infty} \frac{a \tilde{m}^{2}(n)\left(\bar{C}+A_{G} e^{-B_{G} n}\right)}{\left(Q^{2}+\tilde{m}^{2}(n)+A_{m} e^{-B_{m} n}\right)^{3}},
$$

where the notation $\tilde{m}^{2}(n) \equiv \bar{M}^{2}+$ an for the linear part of the $S, P$ trajectory has been introduced. Separating out the first term in the sum and retaining only the leading order in exponential corrections, one obtains

$$
\begin{aligned}
\frac{1}{4} \frac{d^{2} \Pi\left(Q^{2}\right)}{\left(d Q^{2}\right)^{2}} & \simeq \frac{a\left(\bar{C}+A_{G}\right)}{\left(Q^{2}+\bar{M}^{2}+A_{m}\right)^{3}} \\
& +\sum_{n=1}^{\infty}\left\{\frac{a \bar{C} \tilde{m}^{2}(n)}{\left(Q^{2}+\tilde{m}^{2}(n)\right)^{3}}+\frac{a \tilde{m}^{2}(n) A_{G} e^{-B_{G} n}}{\left(Q^{2}+\tilde{m}^{2}(n)\right)^{3}}-\frac{3 a \bar{C} \tilde{m}^{2}(n) A_{m} e^{-B_{m} n}}{\left(Q^{2}+\tilde{m}^{2}(n)\right)^{4}}\right\}
\end{aligned}
$$

In what follows the procedure is the same as for vector channels.

At this point one has to decide which particles are chiral partners in order to guarantee chirally symmetric results. One may think of two possibilities: a) the $\pi$-meson belongs to the radial Regge trajectory being the parity-odd partner of the lightest scalar meson, b) it does not belong to the radial Regge trajectory being an isolated Goldstone chiral particle and therefore the lightest scalar meson is the parity-even partner of the $\pi^{\prime}(1300)$ meson. In case a) the effective low-energy theory would be the linear $\sigma$-model, and in case b) it corresponds to the nonlinear $\sigma$-model. We are going to check both variants. The corresponding sum rules are presented in Appendix B.

\section{Details of fits and results}

In order to compare the sum of the resonances with the OPE we have to choose appropriate values for the inputs of the latter. They are the condensates $\langle\bar{q} q\rangle$ and $\left\langle\left(G_{\mu \nu}^{a}\right)^{2}\right\rangle$, and $\alpha_{s}$. On the resonance side we take as input the pion decay constant $f_{\pi}$, the pion pole residue $Z_{\pi}=2 \frac{\langle\bar{q} q\rangle^{2}}{f_{\pi}^{2}}$ (from current algebra), and the slope $a$. The numerical values for all these parameters are taken at about the CSB scale $\sim 1 \mathrm{GeV}$ and presented in Appendix F. In particular, the value ${ }^{8}$ of $f_{\pi} \approx 103 \mathrm{MeV}$ is certainly different from its low-energy limit [35]

\footnotetext{
${ }^{8}$ This number can be associated to the trade mark of "Brandy de Jerez 103, Osborne"
} 
and corresponds to the matching of the OPE and the sums of resonances at the latter scale (see [37] and references therein).

Recall that in order to keep the discussion in simple terms and also to stay as close as possible to the chiral limit we consider here only non-strange mesons. So far we have also omitted any reference to the isospin degrees of freedom, but we have now to specify the particular pattern in which chiral symmetry is restored in each channel.

In the $V, A$ channels we study isovector states, where the restoration of $U(1)_{A}$ symmetry should be manifest in the large $N_{c}$ limit. We shall therefore analyze the spectra of $\rho$ and $a_{1}$ mesons and their radially excited relatives. In this case five asymptotic sum rules contain seven spectral parameters to be fitted: $M, B_{m, F}, A_{m}^{V, A}$, and $A_{F}^{V, A}$. Thus, we need two additional physical inputs, which are chosen to be the masses of the ground states $\rho$ and $a_{1}$. Actually, we followed the best fit principle when taking the $a_{1}$ mass of about 1200 $\mathrm{MeV}$ in concordance with the masses of first excited vector and axial-vector states. We notice also that the $a_{1}$ mass is very sensitive to the variation of $f_{\pi}$, say, its decrease in 3 $\mathrm{MeV}$ (up to $100 \mathrm{MeV}$ ) leads to diminishing the $a_{1}$ mass to $1180 \mathrm{MeV}$.

Let us comment on some numbers of Table 2 in Appendix F. A possible candidate for the state $m_{V}(2)$ could be the $\rho(1900)$ meson [24]. However, the width of this state is one order of magnitude smaller than the widths of other vector states and it could correspond to a hybrid state (hybrids and glueballs do not lie on the large- $N_{c}$ radial Regge trajectory for quarkonia). Thus, we do not include this meson on the radial Regge trajectory.

Unfortunately, we cannot compare the residues of excited states with experiment. The possible exception is the vector channel due to Eq. (3.4). For example, our ansatz predicts: $\Gamma_{\rho(1450) \rightarrow e^{+} e^{-}}=2.9 \mathrm{KeV}$. The relevant widths $\Gamma_{V \rightarrow e^{+} e^{-}}$are poorly known and not listed in the Particle Data [24]. However, one may compare the results with other independent model estimations. The corresponding numbers we have found in [31]: $\Gamma_{\rho(1450) \rightarrow e^{+} e^{-}}=0.4$ $\mathrm{KeV}$, and in [38]: $\Gamma_{\rho(1450) \rightarrow e^{+} e^{-}}=3.5 \mathrm{KeV}$,

Let us discuss now the D-wave vector mesons. Introducing these states entails the appearance of three new parameters in the asymptotic sum rules: $M_{D}, A_{D}$, and $B_{D}$. The first one can be fixed by the mass of $\rho(1700)$-meson. One can also fix, say $F_{V}(0)$ and $F_{D}(0)$. The average of existing estimates of electromagnetic width for $\rho(1700)$-meson $\left(\Gamma_{\rho(1700) \rightarrow e^{+} e^{-}}=0.1 \mathrm{KeV}[31]\right.$ and $\left.\Gamma_{\rho(1700) \rightarrow e^{+} e^{-}}=2.7 \mathrm{KeV}[38]\right)$ presuppose $^{9}$ a rather large value for $F_{D}(0)$, of the order $60 \mathrm{MeV}$. However, we could not find any reasonable solution for such large values of residue of ground D-wave vector meson. Our estimations showed that $F_{D}(0)$ (i.e., $\left.A_{D}\right)$ must be smaller by about two orders of magnitude than the value of $F_{D}(0)$ obtained from the previous analysis. Then the contribution of D-wave vector mesons to the physical quantities turns out to be below the accuracy of large- $N_{c}$ counting.

\footnotetext{
${ }^{9}$ Strictly speaking, Eq. (3.4) can be applied only to the S-wave vector mesons because of locality of vector current in (3.2). However, in the relativistic theory the situation changes [31] and transitions (3.2) for the D-wave vector states and (3.3) for the axial-vector mesons (which are P-wave states) become possible. Moreover, in the large- $N_{c}$ limit Eqs. (3.2) and (3.3) are well defined even in the relativistic theory since the resonances are narrow. We use (3.4) for the D-wave vector mesons as a rough estimate of coupling these states to the $e^{+} e^{-}$-annihilation.
} 
In the $S, P$ channel the experimental situation is a lot more confusing. In the large $N_{c}$ limit one might equally look for the restoration of $U(1)_{A}$ symmetry relating isotriplet pseudoscalar and scalar states, i.e. pions $(I=1)$ and $a_{0}(I=1)$ mesons and their radial excitations. On the other hand, one could examine the restoration $S U(2)_{L} \times S U(2)_{R}$ symmetry relating pions $(I=1)$ and $f_{0}(I=0)$ in ground and excited states. Both scenarios are compatible if one assumes that there is a degeneracy between $a_{0}$ and $f_{0}$ states. To some extent the latter isoscalar [39] can be related to $f_{0}(980)$ from the PDG data [24]. But for the former isotriplet there is no firm identification with $a_{0}(980)$ from the PDG data [24]. Rather the unitarized fits of pion scattering data [39,40] seem to indicate the dynamical origin of $a_{0}(980)$ as a meson bound state. Thus in order to deal with well established states in the $S, P$ channels in the present paper we have restricted our analysis to asymtotic sum rules relating the isovector pseudoscalar channel (i.e. pions) to the isoscalar meson channel (i.e. $f_{0}$ states). One should of course bear in mind that including strange quarks and moving from $S U(2)$ to $S U(3)$ could bring in some relevant changes in the scalar sector.

In this sector we have considered two possibilities, namely the cases a) and b) at the end of the previous section. Case a) is labeled " $\pi$-in". By this we mean that the pion as well as the lightest scalar meson are taken to be Regge states. We have here seven spectral parameters: $\bar{M}, B_{m, G}, A_{m}^{S, P}$, and $A_{G}^{S, P}$, which are subject to three asymptotic sum rules. As was pointed above, $B_{m}$ is universal for all channels. The three additional conditions are chosen to be: $m_{S}(0)=1 \mathrm{GeV}, m_{P}(0)=0$, and $m_{P}(1)=1.3 \mathrm{GeV}$. This presupposes that the pion and the above scalar are chiral partners. Popular linear $\sigma$-models often require a much lighter scalar with a mass around $600 \mathrm{MeV}$. However our fit favors a heavier scalar quarkonium to provide a realistic value ${ }^{10}$ of $L_{8}$, in agreement with the conclusions of $[39,40]$. The numerical results are presented in Appendix F.

In case b) the $\pi$-meson is assumed not to belong to the radial Regge trajectory being a Goldstone boson that is actually decoupled from the radial Regge trajectory in the CSR limit. This case is labeled " $\pi$-out". According to our fits (the lightest scalar state is again assumed to have a mass of about $1 \mathrm{GeV}$ ), the main qualitative difference between the linear and nonlinear cases is that the first one predicts a scalar meson with a mass of about $1.44 \mathrm{GeV}$ (an iso-singlet resonance with a mass of $1.5 \mathrm{GeV}$ exists but it is widely believed to be a glueball), while the second one does not.

We emphasize again that our analysis in the $S, P$ channels has been performed for $S U(2)$ multiplets including iso-triplet pesudoscalar and iso-singlet scalar mesons. We have also neglected current quark masses adopting the chiral limit. The situation may be drastically different for isotriplet scalars and for $S U(3)$ multiplets. In particular, the $a_{0}(980)$ (isotriplet) mesons may well be dynamical resonances which decouple in the large $N_{c}$ limit whereas the mesons $a_{0}(1450)$ may be dominantly ${ }^{11}$ ground quarkonium states [42]. Their rather large masses could arise owing to a strong mixing between a lighter dynamical scalar $a_{0}$ and a heavier $a_{0}^{\prime}$ quarkonium state with masses of order $1.2 \mathrm{GeV}[41,42]$. We plan to

\footnotetext{
${ }^{10}$ When lowering the input mass of the scalar meson to $600 \mathrm{MeV}$ one gets $L_{8}=2.8 \cdot 10^{-3}$, much higher than the phenomenological estimate $L_{8}=(0.8 \pm 0.3) \cdot 10^{-3}[35]$

${ }^{11}$ We are grateful to the referee who has drawn our attention to this possibility.
} 
investigate this scenario with the help of asymptotic sum rules in a forthcoming paper, taking also into account light quark mass effects.

The quantities $G_{P}(n)$ are related to the corresponding weak decay constants $F_{P}(n)$ through the relation

$$
F_{P}(n)=\frac{2 m_{q} G_{P}(n)}{m_{P}(n)} .
$$

In particular, accepting the average value $m_{q}$ for the current masses of $u$ - and $d$-quarks to be equal to $6 \mathrm{MeV}$, we have the following estimates for the $F_{\pi(1300)}: F_{P}(1)=1.7 \mathrm{MeV}$ (" $\pi$-in") and $F_{P}(0)=1.9 \mathrm{MeV}$ (" $\pi$-out"). Both values are consistent with some previous theoretical predictions $[43,44]$, smaller than the estimates from the Finite-Energy Sum Rules [10] and larger than the predictions from the non-local quark model [45].

It should be also noticed that, allowing for a 10\%-accuracy, the first two chiral pairs of resonances saturate the chiral constant $L_{10}$ introduced in [46] almost completely ${ }^{12}$. But this is not the case for the quantity $\Delta m_{\pi}$ : here, for our fits, one needs to retain about seven pairs. The reason is that the value of this quantity is very sensitive to the violation of the asymptotic sum rules with gluon condensate (strictly speaking, the difference of these sum rules): if one likes to calculate the contribution of the first $N$ pairs of $V, A$ resonances to $\Delta m_{\pi}$, one has then to deal with exactly this number of resonances in the asymptotic sum rules from the very beginning [47].

\section{Summary}

In the present work we have considered the matching of the vector, axial-vector, scalar, and pseudoscalar meson mass spectra $m^{2}(n)$ ( $n$ is the radial quantum number) from Regge theory with universal slope to the Operator Product Expansion of quark currents. The analysis has been carried out for the light non-strange mesons in the large- $N_{c}$ and chiral limits. Let us summarize the important lessons that we gained from our analysis.

- The matching to the OPE cannot be achieved by a simple linear parameterization of the mass spectrum, the linear trajectory ansatz.

- The convergence of the generalized Weinberg sum rules requires the universality of slopes and intercepts for parity conjugated trajectories.

- There must exist deviations from the linear trajectory ansatz triggered by chiral symmetry breaking. These deviations must decrease at least exponentially with $n$.

- There are also deviations from constant residues (decay constants) $F^{2}(n)$ (or for the quantities $G^{2}(n)$ in the scalar case). The analytic structure of OPE imposes again an exponential decrease on these deviations (or faster).

- For heavy states, the D-wave vector mesons have to decouple from asymptotic sum rules. This fact implies the exponential (or faster) decreasing the corresponding decay constants $F_{D}^{2}(n)$.

\footnotetext{
${ }^{12}$ The analytical formulas for the chiral constants $L_{8}, L_{10}$ as well as for $\Delta m_{\pi}$ are given in Appendix $\mathrm{E}$
} 
- Our results seem to exclude a light $\sigma(600)$ particle as a quarkonium state and rather favor the non-linear realization of chiral symmetry with the lightest scalar of mass $\sim 1 \mathrm{GeV}$, its chiral partner being the $\pi^{\prime}(1300)$.

- As a consequence of our approach the quantities $L_{8}, L_{10}$ and $\Delta m_{\pi}$ are obtained, in satisfactory agreement with the phenomenology.

Unfortunately, the underlying dynamics, which generates the non-linear contributions to the spectra of meson masses and residues, is not well known. We can only say that these deviations from the string picture seem to parameterize the chiral symmetry breaking in QCD [4] and, hence, must be proportional to powers of the chiral condensate $\langle\bar{q} q\rangle$ [23]. Developing a theory of these non-linear contributions is an interesting task for future.

Another interesting problem to be examined concerns the $S U(3)$ extension with inclusion of current quark masses into consideration and a possible resolution of the $a_{0}$ meson ambiguity [42]. Finally, one could get some insight by overlapping the present, directresonance approach with spectral density methods [48].

\section{Acknowledgments}

The work of S.A., A.A. and V.A. was supported by the Program "Universities of Russia: Basic Research" (Grant 02.01.016). The work of D.E. was supported by the EURIDICE Network, grant FPA-2001-3598 and grant 2001SGR-00065. All authors enjoyed the support of INTAS-2000 Project 587. A.A. and D.E. wish to thank the warm hospitality of the Pontificia Universidad Catolica de Chile. We are also grateful to A. Pineda and S. Peris for useful discussions.

\section{A. Note on the role of dimension-two condensate}

Let us examine the possibility of having a dimension-two gluon condensate and show its unimportance for fitting meson parameters, at least, in the large- $N_{c}$ limit. The dimensiontwo gluon condensate $\lambda^{2}<0$ ("tachyonic gluon mass") was introduced in [26]. This dimension-two condensate cannot be characterized by a local gauge-invariant operator: for speculations concerning origin, measurement, and physical meaning of this condensate see $[26-28]$.

In $[26,27]$ the following relevant modification of the OPE for quark currents was proposed

$$
\begin{gathered}
\Delta \Pi^{V}\left(Q^{2}\right)=\Delta \Pi^{A}\left(Q^{2}\right)=-\frac{\alpha_{s}}{4 \pi^{3}} \cdot \frac{\lambda^{2}}{Q^{2}} \\
\Delta \Pi^{S}\left(Q^{2}\right)=\Delta \Pi^{P}\left(Q^{2}\right)=-\frac{3 \alpha_{s}}{2 \pi^{3}} \lambda^{2} \ln \frac{\Lambda^{2}}{Q^{2}} .
\end{gathered}
$$

There is no problem to derive such terms from the resonance sums in the $V, A$ channels: their introduction is compatible with the ansätze (4.3), (4.10) (see the corresponding sum

rules in the next Appendix, Eqs. (B.3), (B.4)). However, in order to reproduce the pertinent asymptotical terms in the scalar channels one has to essentially modify the linear part of 
the ansatz (5.1) for the residues of the $S, P$ resonances. We consider two types of possible contributions: an asymptotically constant shift and one affecting only the physical pion residue $(n=0)$,

$$
Z_{S, P}(n) \longrightarrow Z_{S, P}^{\lambda}(n) \equiv 2 G_{S, P}^{2}(n) m_{S, P}^{2}(n)+G_{0}^{\lambda} \frac{d m_{S, P}^{2}(n)}{d n}+\tilde{Z}_{\pi} \delta_{n, 0} .
$$

For this ansatz the appropriate resonance sums (5.3) generate the contribution

$$
\Delta \Pi^{S, P} \simeq G_{0}^{\lambda} \ln \left(\frac{Q^{2}+m^{2}(x)}{\mu^{2}}\right)+\ldots,
$$

which saturates the dimension-two asymptotics (A.2) if

$$
G_{0}^{\lambda}=-\frac{3 \alpha_{s}}{2 \pi^{3}} \lambda^{2}
$$

Then some changes are to be done in the sum rules of the next Appendix.

On the other hand the modification (A.3) evidently affects the pion pole residue $Z_{\pi}$ (at $n=0)$

$$
Z_{\pi} \equiv 2 \frac{\langle\bar{q} q\rangle^{2}}{f_{\pi}^{2}}=\tilde{Z}_{\pi}+a G_{0}^{\lambda}
$$

If we put $\tilde{Z}_{\pi}=0$ and the pion is put on the radial Regge trajectory the required value of the "gluon mass" should be $\lambda^{2} \simeq-2 \mathrm{GeV}^{2}$. However this value is, at least, one order of magnitude higher that any known theoretical estimations [27], $\lambda^{2}=-(0.2 \div 0.5) \mathrm{GeV}^{2}$, and phenomenological bounds (from the analysis of $\tau$-lepton decay) [36], $\lambda^{2}=-(0.05 \pm$ $0.08) \mathrm{GeV}^{2}$.

We conclude that for realistic values of the dimension-two condensate $\lambda^{2}$ its contribution to the residues Eqs. (A.3), (A.6) is negligible. Furthermore one can check that it is certainly less than $5 \%$ for meson masses and decay constants. Still a good open question is about what is a physical observable which is sufficiently sensitive to its presence.

\section{B. Sum rules}

After all summations in (4.15) one arrives at the expansion

$$
-\frac{1}{2} \frac{d \Pi^{V, A}\left(Q^{2}\right)}{d Q^{2}} \simeq \sum_{k=1}^{\infty} \frac{c_{k}^{V, A}}{Q^{2 k}} .
$$

Substituting the expressions for $c_{k}$ and comparing (B.1) with OPE (2.3) and (2.4) we obtain the following asymptotic sum rules in the $V, A$-channels.

At $1 / Q^{2}$

$$
c_{1}^{V, A}=\frac{N_{c}}{24 \pi^{2}}\left(1+\frac{\alpha_{s}}{\pi}\right)=C .
$$

At $1 / Q^{4}$ (the dimension two condensate is taken to be zero as implied by the sum rules in the $S, P$ channels)

$$
c_{2}^{V}=a\left(C+A_{F}^{V}\right)-C\left(\frac{1}{2} a+M^{2}\right)+A_{F}^{V} \Delta_{F}^{(1)}=0,
$$




$$
c_{2}^{A}=a\left(C+A_{F}^{A}\right)-C\left(\frac{1}{2} a+M^{2}\right)+A_{F}^{A} \Delta_{F}^{(1)}=-f_{\pi}^{2}
$$

At $1 / Q^{6}$

$$
\begin{array}{r}
c_{3}^{A}=-2 a\left(C+A_{F}^{V}\right)\left(M^{2}+A_{m}^{V}\right)+C\left(M^{2}\left(M^{2}+a\right)+\frac{1}{6} a^{2}\right)-2 C A_{m}^{V} \Delta_{m}^{(1)}-2 A_{F}^{V} \Delta_{F}^{(2)} \\
=\frac{\alpha_{s}}{12 \pi}\left\langle\left(G_{\mu \nu}^{a}\right)^{2}\right\rangle,
\end{array}
$$

At $1 / Q^{8}$ in $\Pi^{V}\left(Q^{2}\right)-\Pi^{A}\left(Q^{2}\right)$

$$
\begin{aligned}
c_{4}^{V}-c_{4}^{A}=3 a\left(C+A_{F}^{V}\right)\left(M^{2}+A_{m}^{V}\right)^{2}-3 a(C+ & \left.A_{F}^{V}\right)\left(M^{2}+A_{m}^{V}\right)^{2}+6 C\left(A_{m}^{V}-A_{m}^{A}\right) \Delta_{m}^{(2)} \\
& +3\left(A_{F}^{V}-A_{F}^{A}\right) \Delta_{F}^{(3)}=-12 \pi \alpha_{s}\langle\bar{q} q\rangle^{2} .
\end{aligned}
$$

In Eqs. (B.3)-(B.7) the following notations were introduced (the symbol $i$ denotes $m$ or $F$ or $G)$

$$
\begin{gathered}
\Delta_{i}^{(1)}=\frac{a}{e^{B_{i}}-1} \\
\Delta_{i}^{(2)} \equiv \Delta_{i}^{(2)}(M)=\frac{a\left(-M^{2}+\left(M^{2}+a\right) e^{B_{i}}\right)}{\left(e^{B_{i}}-1\right)^{2}}, \\
\Delta_{i}^{(3)} \equiv \Delta_{i}^{(3)}(M)=\frac{a\left[-a\left(a+2 M^{2}\right)+a e^{B_{i}}\left(3 a+2 M^{2}\right)+\left(M^{2}+a\right)^{2}\left(e^{B_{i}}-1\right)^{2}\right]}{\left(e^{B_{i}}-1\right)^{3}} .
\end{gathered}
$$

In the scalar case we can write down analogous to (B.1) expansion:

$$
\frac{1}{4} \frac{d^{2} \Pi^{S, P}\left(Q^{2}\right)}{\left(d Q^{2}\right)^{2}} \simeq \sum_{k=1}^{\infty} \frac{c_{k}^{S, P}}{Q^{2 k}} .
$$

Comparing (B.11) with OPE (2.5) and (2.6) one obtains the asymptotic sum rules. We write them for the $\pi$-out case. The $\pi$-in case can be easily obtained too.

At $1 / Q^{2}$

$$
c_{1}^{S, P}=\frac{N_{c}}{32 \pi^{2}}\left(1+\frac{11 \alpha_{s}}{3 \pi}\right)=\frac{\bar{C}}{2}
$$

At $1 / Q^{6}$

$$
\begin{array}{r}
c_{2}^{S}=a\left(\bar{C}+A_{G}^{S}\right)\left(\bar{M}^{2}+A_{m}^{S}\right)-\frac{\bar{C}}{2}\left(\bar{M}^{2}\left(\bar{M}^{2}+a\right)+\frac{1}{6} a^{2}\right)+A_{G}^{S} \Delta_{G}^{(2)}(\bar{M}) \\
=\frac{\alpha_{s}}{16 \pi}\left\langle\left(G_{\mu \nu}^{a}\right)^{2}\right\rangle
\end{array}
$$




$$
\begin{array}{r}
c_{2}^{P}=\frac{\langle\bar{q} q\rangle^{2}}{f_{\pi}^{2}}+a\left(\bar{C}+A_{G}^{P}\right)\left(\bar{M}^{2}+A_{m}^{P}\right)-\frac{\bar{C}}{2}\left(\bar{M}^{2}\left(\bar{M}^{2}+a\right)+\frac{1}{6} a^{2}\right)+A_{G}^{P} \Delta_{G}^{(2)}(\bar{M}) \\
=\frac{\alpha_{s}}{16 \pi}\left\langle\left(G_{\mu \nu}^{a}\right)^{2}\right\rangle .
\end{array}
$$

At $1 / Q^{8}$ in the $\Pi_{S}\left(Q^{2}\right)-\Pi_{P}\left(Q^{2}\right)$

$$
\begin{array}{r}
c_{3}^{S}-c_{3}^{P}=-3 a\left(\bar{C}+A_{G}^{S}\right)\left(\bar{M}^{2}+A_{m}^{S}\right)^{2}+3 a\left(\bar{C}+A_{G}^{P}\right)\left(\bar{M}^{2}+A_{m}^{P}\right)^{2}-3 \bar{C}\left(A_{m}^{S}-A_{m}^{P}\right) \Delta_{m}^{(2)}(\bar{M}) \\
-3\left(A_{G}^{S}-A_{G}^{P}\right) \Delta_{G}^{(3)}(\bar{M})=-18 \pi \alpha_{s}\langle\bar{q} q\rangle^{2} . \quad(\mathrm{B} .15)
\end{array}
$$

\section{Reference formulae}

The quantities $\Delta_{i}^{(j)}(j=1,2,3$, see (B.8)-(B.10)) appear due to the sums $(B>0)$

$$
\begin{aligned}
\sum_{n=1}^{\infty} e^{-B n} & =\frac{1}{e^{B}-1}, \\
\sum_{n=1}^{\infty} e^{-B n} n & =\frac{e^{B}}{\left(e^{B}-1\right)^{2}}, \\
\sum_{n=1}^{\infty} e^{-B n} n^{2} & =\frac{e^{B}\left(e^{B}+1\right)}{\left(e^{B}-1\right)^{3}} .
\end{aligned}
$$

The summation of the linear in $n$ part is carried out by virtue of the following asymptotic representations (to be precise,once we separated out the first state, one has to make the shift $M^{2} \rightarrow M^{2}+a$ in the expressions below)

$$
\begin{gathered}
\sum_{n=0}^{\infty} \frac{1}{\left(Q^{2}+M^{2}+a n\right)^{2}}=\frac{1}{a^{2}} \psi\left(1, \frac{Q^{2}+M^{2}}{a}\right)=\frac{1}{a}\left\{\frac{1}{Q^{2}}-\frac{1}{Q^{4}}\left(M^{2}-\frac{1}{2} a\right)+\right. \\
\left.\frac{1}{Q^{6}}\left(M^{4}-a M^{2}+\frac{1}{6} a^{2}\right)-\frac{M^{2}}{Q^{8}}\left(M^{2}-\frac{1}{2} a\right)\left(M^{2}-a\right)\right\}+\mathcal{O}\left(\frac{1}{Q^{10}}\right) \\
\sum_{n=0}^{\infty} \frac{M^{2}+a n}{\left(Q^{2}+M^{2}+a n\right)^{3}}=\frac{Q^{2}}{2 a^{3}} \psi\left(2, \frac{Q^{2}+M^{2}}{a}\right)+\frac{1}{a^{2}} \psi\left(1, \frac{Q^{2}+M^{2}}{a}\right)
\end{gathered}
$$

where

$$
\begin{aligned}
& \frac{Q^{2}}{2 a^{3}} \psi\left(2, \frac{Q^{2}+M^{2}}{a}\right)=\frac{1}{a}\left\{-\frac{1}{2 Q^{2}}+\frac{1}{Q^{4}}\left(M^{2}-\frac{1}{2} a\right)-\right. \\
& \left.\frac{3}{2 Q^{6}}\left(M^{4}-a M^{2}+\frac{1}{6} a^{2}\right)+\frac{2 M^{2}}{Q^{8}}\left(M^{2}-\frac{1}{2} a\right)\left(M^{2}-a\right)\right\}+\mathcal{O}\left(\frac{1}{Q^{10}}\right) .
\end{aligned}
$$

Summing Eq. (C.1) and Eq. (C.3) in Eq. (C.2), one can see the absence of term at $1 / Q^{4}$ in the $S, P$-correlators. The corrections contribute only to the following terms, beginning with $1 / Q^{6}$. Thus, in the scalar case the asymptotics corresponding to a dimension two condensate vanishes identically and we do not have a relevant sum rule. 


\section{D-wave vector mesons}

The string model does not take into account the spin of the quarks. This introduces two type of states depending on the relative angular momentum. In short, there exist S-wave and D-wave $\rho$-mesons and this results in doubling of the $\rho$-meson trajectory $[1,2]$. Although conceptually simple, this complicates the analysis considerably.

The two kinds of $\rho$-mesons should enter the sum rules independently. This observation, generally speaking, leads to the constrain in Eq. (3.9)

$$
\frac{F_{V}^{2}(n)}{a_{V}}+\frac{F_{D}^{2}(n)}{a_{D}}=\frac{F_{A}^{2}(n)}{a_{A}}, \quad n \rightarrow \infty
$$

where the label $D$ stands for the D-wave $\rho$-mesons. It is seen from numerical estimations (especially for the correct ansatz $a_{V}=a_{D}=a$ ) that $F_{D}(n)$ must be very small compared with $F_{V}(n)$. This follows also from consideration of the matrix element $\left\langle 0\left|\bar{q} \gamma_{\mu} q\right| \rho^{0}\right\rangle$. It is clear qualitatively that the D-wave final state is strongly suppressed compared with the Sone, although a concrete numerical answer will depend on how one models the wave function of $\rho^{0}$-meson. In the non-relativistic case this statement is trivial: S-wave component of wave function behaves as a constant and D-one does as $r^{2}$ at small distances $r$. Hence the latter tends to zero since annihilation is a point-like process. Thus, D-wave $\rho$-mesons, at least asymptotically, should drop out from the sum rules in contrast to what has been stated in [19], where this doubling was examined by means of quasi-classical string analysis.

Let us consider the linear ansatz for the mass spectrum of D-wave vector mesons

$$
m_{D}^{2}(n)=M_{D}^{2}+a n .
$$

Direct insertion of this ansatz into sum rules (4.6) would automatically imply by the CSR that $m_{D}=M$. This contradicts phenomenology [1,2]: S- and D-trajectories seem to have a constant splitting at any energy. In order to satisfy the sum rules of type (4.6), the D-wave residues should decrease at least exponentially. We propose the following parameterization

$$
F_{D}^{2}(n)=a A_{D} e^{-B_{D} n}, \quad B_{D}>0 .
$$

As these residues are exponentially small we do not consider the exponential corrections to the mass spectrum of D-wave $\rho$-mesons (D.2) in our approximation.

There arises the question as to how the chiral limit affects the behavior of the D-wave vector trajectory. One may think of two possibilities: i) the $\mathrm{D}$-wave mesons approach the $\mathrm{S}$-wave vector trajectory, implying asymptotic degeneration; ii) D-wave $\rho$-mesons decouple. The latter variant seems to be more plausible since the former one signifies the doubling of states on the vector trajectory compared with the axial-vector one. In addition, the decoupling must take place in any case by virtue of the qualitative arguments mentioned above.

\section{E. Electromagnetic pion mass difference $\Delta m_{\pi}$ and chiral constants $L_{8}, L_{10}$.}

Given an ansatz for mass spectrum $m_{J}^{2}(n)$ and decay constants $F_{J}^{2}$, one can calculate the electromagnetic pion mass difference $\Delta m_{\pi} \equiv m_{\pi^{+}}-m_{\pi^{0}}$, the chiral constant $L_{10}$ [46] 
(parameterizing the decay $\pi \rightarrow e \nu \gamma$ ), and a $K \rightarrow \pi$ matrix element of the electromagnetic penguin operator $Q_{7}^{3 / 2}$. An example of such a calculation for the LTA was provided in [16]. Besides, in the scalar sector we can calculate the chiral constant $L_{8}$ [46] (parameterizing the ratio of current quark masses). We did not consider the operator $Q_{7}^{3 / 2}$ since this quantity strongly depends on the cutoff and is of a limited phenomenological interest. The other quantities are determined by

$$
\begin{gathered}
L_{10}=-\frac{1}{8} \frac{d}{d Q^{2}}\left[Q^{2}\left(\Pi^{V}\left(Q^{2}\right)-\Pi^{A}\left(Q^{2}\right)\right)\right]_{Q^{2}=0}, \\
\Delta m_{\pi}=-\frac{3 \alpha}{16 \pi m_{\pi} f_{\pi}^{2}} \int_{0}^{\infty} d Q^{2} Q^{2}\left(\Pi^{V}\left(Q^{2}\right)-\Pi^{A}\left(Q^{2}\right)\right), \\
L_{8}=\frac{f_{\pi}^{4}}{32\langle\bar{q} q\rangle^{2}} \frac{d}{d Q^{2}}\left[Q^{2}\left(\Pi^{S}\left(Q^{2}\right)-\Pi^{P}\left(Q^{2}\right)\right)\right]_{Q^{2}=0} .
\end{gathered}
$$

In our approach, one can directly sum over all resonances in this quantities due to the exact cancellation of divergent parts. Making use of notations (4.10),(4.13) and (D.2) one can write for (E.1)-(E.3) the convergent sums (we pick out the ground states, treat them exactly and retain only the leading and linear in exponential terms for the other ones)

$$
\begin{aligned}
& L_{10}=\frac{a}{4}\left\{\frac{C+A_{F}^{A}}{M^{2}+A_{m}^{A}}-\frac{C+A_{F}^{V}}{M^{2}+A_{m}^{V}}\right. \\
& \left.+\sum_{n=1}^{\infty} \frac{\bar{m}^{2}(n) e^{-B_{F} n}\left(A_{F}^{A}-A_{F}^{V}\right)-C e^{-B_{m} n}\left(A_{m}^{A}-A_{m}^{V}\right)}{\bar{m}^{4}(n)}\right\}, \\
& \Delta m_{\pi}=\frac{3 \alpha a}{16 \pi m_{\pi} f_{\pi}^{2}}\left\{\left(C+A_{F}^{A}\right)\left(M^{2}+A_{m}^{A}\right) \ln \frac{M^{2}+A_{m}^{A}}{\mu^{2}}\right. \\
& -\left(C+A_{F}^{V}\right)\left(M^{2}+A_{m}^{V}\right) \ln \frac{M^{2}+A_{m}^{V}}{\mu^{2}}+\sum_{n=1}^{\infty}\left(C \bar{m}^{2}(n) \ln \frac{m_{A}^{2}(n)}{m_{V}^{2}(n)}\right. \\
& \left.\left.+\left[C e^{-B_{m} n}\left(A_{m}^{A}-A_{m}^{V}\right)+\bar{m}^{2}(n) e^{-B_{F} n}\left(A_{F}^{A}-A_{F}^{V}\right)\right] \ln \frac{\bar{m}^{2}(n)}{\mu^{2}}\right)\right\} .
\end{aligned}
$$

In the $\pi$-in case

$$
\begin{array}{r}
L_{8}=\frac{f_{\pi}^{4} a}{16\langle\bar{q} q\rangle^{2}}\left\{\bar{C}+A_{G}^{S}+\sum_{n=1}^{\infty} e^{-B_{G} n}\left(A_{G}^{S}-A_{G}^{P}\right)\right\}= \\
\frac{f_{\pi}^{4} a}{16\langle\bar{q} q\rangle^{2}}\left\{\bar{C}+A_{G}^{S}+\frac{\left(A_{G}^{S}-A_{G}^{P}\right)}{e^{B_{G}}-1}\right\},
\end{array}
$$

while for the $\pi$-out case

$$
L_{8}=\frac{f_{\pi}^{4} a}{16\langle\bar{q} q\rangle^{2}} \sum_{n=0}^{\infty} e^{-B_{G} n}\left(A_{G}^{S}-A_{G}^{P}\right)=\frac{f_{\pi}^{4} a}{16\langle\bar{q} q\rangle^{2}} \frac{\left(A_{G}^{S}-A_{G}^{P}\right)}{1-e^{-B_{G}}} .
$$

The parameter $\mu$ in Eq. (E.5) is a normalization scale. The result does not depend on it as it is seen from the difference of sum rules (B.5) and (B.6).

Accepted estimates for $L_{10}$ and $L_{8}$ from phenomenology are: $\left.L_{10}\right|_{\text {phen }}=(-5.5 \pm 0.7) \cdot 10^{-3}[35]$ and $\left.L_{8}\right|_{\text {phen }}=(0.8 \pm 0.3) \cdot 10^{-3}[35]$. 


\section{F. Numerical analysis}

In this Appendix we give an example of the meson mass spectra resulting from our work. The inputs general for all tables (if any) are: $a=(1120 \mathrm{MeV})^{2},\langle\bar{q} q\rangle=-(240 \mathrm{MeV})^{3}$, $\frac{\alpha_{s}}{\pi}\left\langle\left(G_{\mu \nu}^{a}\right)^{2}\right\rangle=(360 \mathrm{MeV})^{4}, f_{\pi}=103 \mathrm{MeV}, Z_{\pi}=2 \frac{\langle\bar{q} q\rangle^{2}}{f_{\pi}^{2}}, \alpha_{s}=0.3$. The units are: $m(n)$, $F(n), G(n)-\mathrm{MeV} ; A_{m}-\mathrm{MeV}^{2} ; A_{F}, A_{G}, B_{F, G, m}-\mathrm{MeV}^{0}$.

\begin{tabular}{|c||l|l|}
\hline Case & \multicolumn{1}{|c|}{ Inputs } & \multicolumn{1}{c|}{ Fits and constants } \\
\hline \hline \multirow{4}{*}{$V A$} & & $M=920, B_{m}=0.97, B_{F}=0.72$, \\
& $m_{V}(0)=770(769.3 \pm 0.8)$, & $A_{m}^{V}=-500^{2}, A_{m}^{A}=770^{2}$, \\
& $m_{A}(0)=1200(1230 \pm 40)$, & $A_{F}^{V}=0.0012, A_{F}^{A}=-0.0031$, \\
& & $L_{10}=-6.5 \cdot 10^{-3}, \Delta m_{\pi}=2.3$ \\
\hline \hline \multirow{3}{*}{$S P$} & $m_{S}(0)=1000$, & $\bar{M}=840, B_{G}=0.42$, \\
$(\pi$-in $)$ & $m_{P}(0)=0$, & $A_{m}^{S}=550^{2}, A_{m}^{P}=-840^{2}$, \\
& $m_{P}(1)=1300(1300 \pm 100)$, & $A_{G}^{S}=-0.0009, A_{G}^{P}=0.0004$, \\
& $B_{m}=0.97$ & $L_{8}=1.0 \cdot 10^{-3}$ \\
\hline \hline \multirow{3}{*}{$S P$} & $m_{S}(0)=1000$, & $\bar{M}=1470, B_{G}=1.27$, \\
$(\pi$-out $)$ & $m_{P}(0)=1300(1300 \pm 100)$, & $A_{m}^{S}=-1080^{2}, A_{m}^{P}=-690^{2}$, \\
& $m_{P}(1)=1800(1801 \pm 13)$, & $A_{G}^{S}=0.0213, A_{G}^{P}=0.0067$, \\
& $B_{m}=0.97$ & $L_{8}=0.9 \cdot 10^{-3}$ \\
\hline
\end{tabular}

Table 1: An example of parameters for the mass spectra of our work. The corresponding experimental values $[2,24]$ (if any) are displayed in brackets. 


\begin{tabular}{|c||l|l|l|l|l|}
\hline$n$ & out & \multicolumn{1}{c|}{0} & \multicolumn{1}{c|}{1} & \multicolumn{1}{c|}{3} \\
\hline \hline$m_{V}(n)$ & & $770(769.3 \pm 0.8)$ & $1420(1465 \pm 25)$ & 1820 & $2140(2149 \pm 17)$ \\
$F_{V}(n)$ & & $138(154 \pm 8)$ & 135 & 133 & 133 \\
\hline$m_{A}(n)$ & & $1200(1230 \pm 40)$ & $1520(1640 \pm 40)$ & $1850(1971 \pm 15)$ & $2150(2270 \pm 50)$ \\
$F_{A}(n)$ & & $116(123 \pm 25)$ & 125 & 128 & 130 \\
\hline \hline$m_{S}(n)$ & & $1000(980 \pm 10)$ & 1440 & $1800(1713 \pm 6)$ & 2100 \\
$G_{S}(n)$ & & 176 & 178 & 178 & 179 \\
\hline$m_{P}(n)$ & & 0 & $1300(1300 \pm 100)$ & $1760(1801 \pm 13)$ & $2100(2070 \pm 35)$ \\
$G_{P}(n)$ & & - & 179 & 179 & 179 \\
\hline \hline$m_{S}(n)$ & & $1000(980 \pm 10)$ & $1730(1713 \pm 6)$ & 2120 & 2420 \\
$G_{S}(n)$ & & 243 & 199 & 185 & 181 \\
\hline$m_{P}(n)$ & 0 & $1300(1300 \pm 100)$ & $1800(1801 \pm 13)$ & $2150(2070 \pm 35)$ & $2430(2360 \pm 30)$ \\
$G_{P}(n)$ & - & 201 & 186 & 181 & 180 \\
\hline
\end{tabular}

Table 2: Mass spectrum and residues for the parameter sets of Table 1. The known experimental values $[2,24]$ are displayed in brackets. Not all the masses of scalar mesons are related to experimental ones since their correspondence is not well established yet because of strong mixing and unitarization effects. When comparing the predicted masses to the physical mass spectrum no attempt has been made to correct for the non-zero quark mass other than for the pion. Except for the pion the relative effect of quark masses is very small.

\section{References}

[1] A.V. Anisovich, V.V. Anisovich and A.V. Sarantsev, Phys. Rev. D 62 (2000) 051502.

[2] V.V. Anisovich, hep-ph/0208123.

[3] G. Veneziano, Nuovo Cim. A57 (1968) 190.

[4] J. Alfaro, A.A. Andrianov, L.Balart and D. Espriu, Int. J. Mod. Phys. A 18 (2003) 2501; A.A. Andrianov and D. Espriu, Theor. Math. Phys. 135 (2003) 745.

[5] A. Bramon, E. Etim and M. Greco, Phys. Lett. B 41 (1972) 609;

M. Greco, Nucl. Phys. B 63 (1973) 398; J.J. Sakurai,Phys. Lett. B 46 (1973) 207.

[6] G. 't Hooft, Nucl. Phys. B 72 (1974) 461; E. Witten, Nucl. Phys. B 160 (1979) 57.

[7] A.R. Zhitnitsky, Phys. Rev. D 53 (1996) 5821;

B. Blok, Nucl. Phys. 64 (Proc. Suppl.) (1998) 481;

B. Blok, M. Shifman and D-X. Zhang, Phys. Rev. D 57 (1998) 2691; Erratum, Phys. Rev. D 59 (1999) 019901.

[8] E.C. Poggio, H.R. Quinn and S. Weinberg, Phys. Rev. D 13 (1976) 1958.

[9] N.V. Krasnikov, A.A. Pivovarov, Yad. Fiz. 35 (1982) 1270; Phys. Lett. B 112 (1982) 397.

[10] A.L. Kataev, N.V. Krasnikov, A.A. Pivovarov, Phys. Lett. B 123 (1983) 93.

[11] S.G. Gorishnii, A.L. Kataev, S.A. Larin, Phys. Lett. B 135 (1984) 457. 
[12] E.A. Tainov, Z. Physik C 10 (1981) 87;

B.V. Geshkenbein, Yad. Fiz. 42 (1985) 991.

[13] B.V. Geshkenbein, Sov. J. Nucl. Phys. 49 (1989) 705.

[14] M.A. Shifman, A.I. Vainstein and V.I. Zakharov, Nucl. Phys. B 147 (1979) 385, 448.

[15] M. Shifman, At the Frontier of Particle Physics/Handbook of QCD, (ed. M. Shifman), World Scientific, 2001; hep-ph/0009131.

[16] M. Golterman and S. Peris, J. High Energy Phys. 01 (2001) 028.

[17] S.R. Beane, Phys. Rev. D 64 (2001) 116010.

[18] S.R. Beane, Phys. Lett. B 521 (2001) 47.

[19] Yu.A. Simonov, Phys. Atom. Nucl. 65 (2002) 135; hep-ph/0109081.

[20] M. Golterman, S. Peris, B. Phily and E. de Rafael, J. High Energy Phys. 01 (2002) 024.

[21] M. Golterman and S. Peris, Phys. Rev. D 67 (2003) 096001.

[22] A.A. Andrianov, V.A. Andrianov and S.S. Afonin, Proceedings of the 5th Int. Conf. on Quark Confinement and the Hadron Spectrum, Gargnano (Italy), 2002, World Scientific, 2003, p.361; hep-ph/0212171.

[23] S.S. Afonin, Phys. Lett. B 576 (2003) 122.

[24] Particle Date Group: K. Hagiwara et al., Phys. Rev. D 66 (2002) 010001.

[25] L.J. Reinders, S. Yazaki and H.R. Rubinstein, Nucl. Phys. B 196 (1982) 125.

[26] K.G. Chetyrkin, S. Narison and V.I. Zakharov, Nucl. Phys. B 550 (1999) 353.

[27] F.V. Gubarev and V.I. Zakharov, Phys. Lett. B 501 (2001) 28;

S. Narison and V.I. Zakharov, Phys. Lett. B 522 (2001) 266.

[28] K.I. Kondo, Phys. Lett. B 572 (2003) 210;

K.I. Kondo, T. Murakami, T. Shinohara and T. Imai, Phys. Rev. D 65 (2002) 085034.

[29] A.A. Andrianov, D. Espriu and R. Tarrach, Nucl. Phys. B 533 (1998) 429; Nucl. Phys. 86 (Proc. Suppl.) (2000) 275.

[30] M. Knecht and E. de Rafael, Phys. Lett. B 424 (1998) 335; A.A. Andrianov, V.A. Andrianov, Proc. Int. Worksh. on Hadron Physics Coimbra, 1999; AIP 508 (2000) 382; hep-ph/9911383.

[31] S. Godfrey and N. Isgur, Phys. Rev. D 32 (1985) 189.

[32] G. Ecker, J. Gasser, A. Pich and E. de Rafael, Nucl. Phys. B 321 (1989) 311.

[33] S. Weinberg, Phys. Rev. Lett. 18 (1967) 507;

C.W. Bernard, A. Duncan, J. LoSecco and S. Weinberg, Phys. Rev. D 12 (1975) 792.

[34] M.A. Shifman, A.I. Vainstein and V.I. Zakharov, Sov. Phys. JETP Lett. 27 (1978) 55; A.A. Andrianov, V.A. Andrianov, V.Yu. Novozhilov and Yu.V. Novozhilov, Phys. Lett. B 186 (1987) 401;

A.A. Andrianov and V.A. Andrianov, Z. Physik C 55 (1992) 435.

[35] A. Pich, Rept. Prog. Phys. 58 (1995) 563; hep-ph/9502366. 
[36] C.A. Dominguez and K. Schilcher, Phys. Rev. D 61 (2000) 114020;

B.V. Geshkenbein, B.L. Ioffe and K.N. Zyablyuk, Phys. Rev. D 64 (2001) 093009

[37] M. Harada and K. Yamawaki, Phys. Rept. 381 (2003) 1.

[38] V.K. Henner and T.S. Belozerova, Phys. Atom. Nucl. 59 (1996) 1851; Phys. Atom. Nucl. 61 (1998) 119.

[39] J.A. Oller and E. Oset, Phys. Rev. D 60 (1999) 074023

[40] J.R. Peláez, AIP 687 (2003) 74; hep-ph/0306063; Phys. Rev. Lett. 92 (2004) 102001.

[41] D. Black, A.H. Fariborz and J. Schechter, Phys. Rev. D 61 (2000) 074001; hep-ph/0008246.

[42] V. Cirigliano, G. Ecker, H. Neufeld and A. Pich, J. High Energy Phys. 06 (2003) 012.

[43] C.A. Dominguez, Phys. Rev. D 15 (1977) 1350; ibid. 16 (1977) 2313, 2320.

[44] A.A. Andrianov, V.A. Andrianov and A.N. Manashov, Int. J. Mod. Phys. A 6 (1991) 5435;

V. Elias, A. Fariborz, M.A. Samuel, Fang Shi and T.G. Steele, Phys. Lett. B 412 (1997) 131.

[45] M.K. Volkov and C. Weiss, Phys. Rev. D 56 (1997) 221.

[46] J. Gasser and H. Leutwyler, Nucl. Phys. B 250 (1985) 465.

[47] V.A. Andrianov and S.S. Afonin, Phys. Atom. Nucl. 65 (2002) 1862; hep-ph/0109026.

[48] S. Groote, J.G. Körner and A.A. Pivovarov, Phys. Rev. D 65 (2002) 036001. 